\title{
Robust Control of Polytopic Systems by Convex Optimization
}

\author{
Alireza Karimi, Hamid Khatibi and Roland Longchamp
}

\begin{abstract}
Robust control synthesis of linear time-invariant SISO polytopic systems is investigated using the polynomial approach. A convex set of all stabilizing controllers for a polytopic system is given over an infinite-dimensional space. A finite-dimensional approximation of this set is obtained using the orthonormal basis functions and represented by a set of LMIs thanks to the KYP lemma. Then, an LMI based convex optimization problem for robust pole placement with sensitivity function shaping in two- and infinity-norm is proposed. The simulation results show the effectiveness of the proposed method.
\end{abstract}

\section{INTRODUCTION}

One of the most general ways to describe without any conservatism the physical parameter uncertainty is the polytopic representation. In this representation, the uncertain system belongs to a polytope which is the convex hull of the parameters of a set of models (vertices). Polytopic uncertainty can cover the well-known interval and linear parameter uncertainty as well as multi-model structures.

Robust stability of polytopic systems is analyzed either in the polynomial form using the generalized Kharitonov theorem [1] or in the state space using the concept of quadratic stability. In the latter, a Lyapunov function should be found for all vertices of the polytopic system. However, this approach suffers considerably from conservatism [2]. A less conservative approach is the use of a parameter dependent Lyapunov function which leads to a sufficient condition for the robust stability represented by LMIs [3], [4], [5].

Although robust stability analysis for polytopic systems has been adequately studied in the literature, the results for robust control synthesis of these systems are rather limited. A classical approach is $\mu$ synthesis which leads to a nonconvex optimization problem [6]. The convex optimization solutions are generally based on the quadratic stability of the whole polytope using a Lyapunov function which turns out to be too conservative. In most of the early approaches, a state feedback controller is designed based on the parameter dependent Lyapunov approach and the use of convex optimization algorithms via LMIs [7], [8]. To the best of our knowledge, the only approaches that do not consider the quadratic stability for the output feedback design of polytopic systems and use a convex optimization algorithm

\footnotetext{
This paper has been provisionally accepted for Automatica but has never been presented in a conference.

The authors are with the Laboratoire d'Automatique of Ecole Polytechnique Fédérale de Lausanne (EPFL), 1015 Lausanne, Switzerland.

Supported by the Swiss National Science Foundation under Grant No. 200021-100379.

Corresponding author: alireza.karimi@epfl.ch
}

are given by Rantzer and Megretski in [9] and Henrion et al. in [10]. Rantzer and Megretski propose a synthesis method which can consider a specific "rank one" type of uncertainty that covers the polytopic uncertainty with some conservatism. Moreover, since a Q-parameterization method is involved in the synthesis approach, fixed-order controller design cannot be handled. In [10] a convex parameterization of fixed-order controllers for polytopic systems based on the polynomial positivity is given. The approach gives only a stabilizing controller (feasible point) which relies on a central polynomial whose choice is not studied.

The main idea of this paper comes from the problem of robust strict positive realness of transfer functions. This problem has been considered by many authors [11], [12], [13], [14]. The necessary and sufficient conditions are studied for the existence and construction of a polynomial or transfer function $d$ which makes $p / d$ strictly positive real for all $p$ in a convex set of real polynomials. These results are used to propose a convex set (in an infinite dimensional space) of all stabilizing controllers for a polytopic system. For fixed-order controllers, an inner approximation of this set is obtained based on LMIs from the Kalman-Yakubovic-Popov lemma (KYP). Furthermore, the pole placement and sensitivity function shaping [15], [16] are considered as performance specifications to design controllers assuring robust stability and robust performance for SISO LTI polytopic systems.

The contributions of this paper with respect to the method in [10] are as follows: A convex set of all stabilizing controllers for a polytopic system is given; The inner approximation of this convex set for fixed-order controllers is the same as that in [10] but is based on the KYP lemma instead of the positive polynomials; The convex functions and constraints for robust performance of the polytopic systems and robust stability with respect to unstructured uncertainty are introduced. The pole placement technique gives the guidelines for selecting the central polynomial which was a challenging step in [10].

The rest of the paper is organized as follows. A new convex parameterization of all stabilizing controllers for the polytopic system is given in Section II. The performance specifications in terms of combined pole placement with sensitivity function shaping in $\mathcal{H}_{2}$ or $\mathcal{H}_{\infty}$ norm are introduced in Section III. Section IV shows how the proposed convex optimization problem can be represented by LMIs. The simulation results in Section V illustrate the effectiveness of the proposed synthesis method. Finally, concluding remarks are given in Section VI. 


\section{Convex Parameterization}

Consider a SISO LTI plant represented by a finite order rational transfer function $G$ in discrete- or in continuoustime. Assume that $N$ and $M$ are the coprime factors of $G$ such that

$$
G=N M^{-1}, \quad N, M \in \mathcal{R} \mathcal{H}_{\infty}
$$

where $\mathcal{R} \mathcal{H}_{\infty}$ is the set of proper stable rational transfer functions with bounded infinity norm. Consider also the convex set of all strictly positive real transfer functions $\mathcal{S}$. The transfer functions in $\mathcal{S}$ are stable and inversely stable with a strictly positive real part on the boundary of the stability domain.

The objective is to give an alternative to the well-known Youla parameterization technique for convex parameterization of all stabilizing controllers. The main advantage of the new parameterization is that it can be easily extended to the multi-model case and systems with real parameter uncertainty. The following theorem introduces this parameterization.

Theorem 1: The set of all stabilizing controllers for $G$ defined in (1) is given by:

$$
\mathcal{K}:\left\{K=X Y^{-1} \mid M Y+N X \in \mathcal{S}\right\}
$$

where $X, Y \in \mathcal{R H}_{\infty}$.

Proof Sufficiency: It is clear that the zeros of $M Y+N X$ include the closed-loop poles and because $M Y+N X$ is SPR, its zeros are also inside the stability region.

Necessity: We should show that if $K_{0}$ is a stabilizing controller then it belongs to $\mathcal{K}$. Suppose that $K_{0}=X_{0} Y_{0}^{-1}$ where $X_{0}, Y_{0} \in \mathcal{R} \mathcal{H}_{\infty}$ are its coprime factors. Then, $M Y_{0}+$ $N X_{0}$ is biproper, stable and inversely stable. For such a system there always exists a transfer function $F \in \mathcal{R} \mathcal{H}_{\infty}$ such that $\left(M Y_{0}+N X_{0}\right) F$ is SPR (for instance taking $F$ equal to the inverse of $M Y_{0}+N X_{0}$ ). Hence, $K_{0}$ belongs to $\mathcal{K}$ with $X=X_{0} F$ and $Y=Y_{0} F$.

It should be mentioned that the set $\mathcal{K}$ can be represented by a set of convex constraints in the pair $(X, Y)$ as follows:

$$
\operatorname{Re}[M(j \omega) Y(j \omega)+N(j \omega) X(j \omega)]>0, \quad \forall \omega \in \mathcal{R} \cup\{\infty\}
$$

$X$ and $Y$ can be approximated using different types of orthonormal basis functions like Laguerre, Kauts or a generalized basis function introduced in [17]. For instance, consider that $X$ and $Y$ are linearly parameterized by:

$$
X=\sum_{i=1}^{m} x_{i} \beta_{i} \quad ; \quad Y=\sum_{i=1}^{m} y_{i} \beta_{i}
$$

where $\beta_{i}, i=1, \ldots, m$ are the basis functions. As a result, the constraints in (3) become linear in the parameters of $X$ and $Y$ and can be represented by an LMI using the KYP lemma (see Section IV).

The main feature of this parameterization is that it can be applied to the systems with polytopic uncertainty. Consider a polytopic system with $q$ vertices such that the $i$-th vertex constitutes the parameters of the model $G_{i}=N_{i} M_{i}^{-1}$ where
$N_{i}$ and $M_{i} \in \mathcal{R} \mathcal{H}_{\infty}$ are the coprime factors of $G_{i}$. Also without loss of generality, assume that the denominators of $N_{i}$ and $M_{i}$ are fixed and are the same for $i=1, \ldots, q$, i.e. the model parameters appear linearly in the numerators of $N_{i}$ and $M_{i}$. The set of all models in this polytopic system can be shown by:

$$
\mathcal{G}:\left\{G=N M^{-1} \mid N=\sum_{i=1}^{q} \lambda_{i} N_{i}, M=\sum_{i=1}^{q} \lambda_{i} M_{i}\right\}
$$

where $\lambda_{i} \geq 0$ and $\sum_{i=1}^{q} \lambda_{i}=1$.

The aim is to parameterize all stabilizing controllers for this polytopic system. The controller parameterization is given in the following theorem:

Theorem 2: The set of all stabilizing controllers for the polytopic system defined in (5) is given by:

$$
\mathcal{K}:\left\{K=X Y^{-1} \mid M_{i} Y+N_{i} X \in \mathcal{S}, \quad i=1, \ldots, q\right\}
$$

where $X, Y \in \mathcal{R H}_{\infty}$.

Proof Sufficiency: It should be shown that if $M_{i} Y+N_{i} X$ is SPR for $i=1, \ldots, q$, then the controller $K=X Y^{-1}$ stabilizes all the elements of the polytopic system $\mathcal{G}$. Since $M_{i} Y+N_{i} X \in \mathcal{S}$ for $i=1, \ldots, q$ and the set $\mathcal{S}$ is convex, one obtains

$$
\sum_{i=1}^{q} \lambda_{i}\left[M_{i} Y+N_{i} X\right] \in \mathcal{S}, \quad \lambda_{i} \geq 0, \quad \sum_{i=1}^{q} \lambda_{i}=1
$$

Therefore

$$
\left(\sum_{i=1}^{q} \lambda_{i} M_{i}\right) Y+\left(\sum_{i=1}^{q} \lambda_{i} N_{i}\right) X \in \mathcal{S}
$$

and $M Y+N X$ is SPR. Hence, the zeros of $M Y+N X$ (the closed-loop poles) are inside the stability domain for any model in the polytopic system $\mathcal{G}$.

Necessity: Assume that $K_{0}=X_{0} Y_{0}^{-1}$ stabilizes the polytopic system $\mathcal{G}$. Then, a polytope of stable characteristic polynomials with the vertices $c_{i}^{0}$ can be constructed from the controller $K_{0}$ and the vertices $G_{i}$ of the polytopic system. It has been shown that for such a polytope the phase difference between its elements is less than $\pi$ [1]. Therefore, according to Theorem 2.1 (for discrete-time systems) and Theorem 3.1 (for continuous-time systems) in [11] there always exists a polynomial or transfer function $d$ such that $c_{i}^{0} / d, i=1, \ldots, q$ are SPR transfer functions. As a result, there exists a transfer function:

$$
F=\left(M_{i} Y_{0}+N_{i} X_{0}\right)^{-1} c_{i}^{0} / d \in \mathcal{R} \mathcal{H}_{\infty}
$$

such that $\left(M_{i} Y_{0}+N_{i} X_{0}\right) F$ for $i=1, \ldots, q$ is SPR. Note that the numerator of $\left(M_{i} Y_{0}+N_{i} X_{0}\right)$ is equal to $c_{i}^{0}$ and is canceled in (9). Hence, $K_{0}$ belongs to $\mathcal{K}$ with $X=X_{0} F$ and $Y=Y_{0} F$.

\section{Remarks}

- An interesting property of this parameterization is that it can be used straightforwardly for the low-order controller design. In other words, for a finite $m$, the 
constraints in (3) give an inner convex approximation of all $m$-th order stabilizing controllers and when $m$ tends to infinity this set converges to the set of all stabilizing controllers for the polytopic system $\mathcal{G}$.

- Although the proposed parameterization has no conservatism for stabilizing the polytopic systems, it suffers from conservatism when applied to multi-model uncertainty because a whole polytope is stabilized instead of only the vertices.

- For the stable systems with multi-model uncertainty, the set of stabilizing controllers can be presented by (choosing $N_{i}=G_{i}$ and $M_{i}=1$ ):

$$
\mathcal{K}:\left\{K=X Y^{-1} \mid Y+G_{i} X \in \mathcal{S}, i=1, \ldots, q\right\}
$$

This set includes the controllers that stabilize all convex combinations of $G_{i}$, i.e. $\sum_{i} \lambda_{i} G_{i}$ such that $\sum_{i} \lambda_{i}=1$ and $\lambda_{i} \geq 0$. Note that this convex set is not appropriate for polytopic systems since the model parameters appear nonlinearly in the constraints. However, it may be used for systems with frequency domain uncertainty.

\section{PERformance SPECificAtions}

In the previous section, a convex parameterization of all stabilizing controllers for the polytopic systems was introduced. Here, different convex optimization problems are defined to ensure the robust performance for the polytopic systems. First, a technique for robust pole placement is introduced. Then it is shown how this method can be combined with sensitivity function shaping to improve the closed-loop performance.

\section{A. Pole Placement}

Consider first a simple case where only a model $G$ is available and the objective is to assign the closed-loop poles at the desired positions using a convex optimization problem. To proceed, the coprime factors $N$ and $M$ of $G$ are computed such that the roots of their denominators contain the desired dominant closed-loop poles. Then, $X$ and $Y$ are approximated by orthonormal basis functions. Here, suppose that the system is in discrete-time and the so-called Laguerre model is used. In this model the basis functions are:

$$
\beta_{i}(z)=\left(\frac{\sqrt{1-\xi^{2}}}{z-\xi}\right)\left(\frac{1-\xi z}{z-\xi}\right)^{i}
$$

where $\xi$ is the position of desired auxiliary closed-loop poles (chosen faster than the dominant ones). With this choice, the roots of the denominator of $M Y+N X$ are the desired closed-loop poles and its numerator is the closedloop characteristic polynomial. Now, consider the following convex optimization problem:

$$
\begin{aligned}
& \text { Minimize }\|M Y+N X-1\| \\
& \text { Subject to: } \\
& M Y+N X \in \mathcal{S}
\end{aligned}
$$

where $\|\cdot\|$ can be any transfer function norm. It is clear that if the order of $X$ and $Y$ is properly chosen such that the controller that assigns the closed-loop poles in the desired places belongs to the set of parameterized controllers, then this controller is the solution to the above optimization problem and makes the objective function equal to zero. However, for a lower order controller an approximate pole placement will be achieved.

The proposed technique can be extended to the polytopic system $\mathcal{G}$ using the following convex optimization problem:

\section{Minimize $\gamma$}

Subject to:

$$
\begin{array}{ll}
\left\|M_{i} Y+N_{i} X-1\right\|<\gamma & \text { for } i=1, \ldots, q \\
M_{i} Y+N_{i} X \in \mathcal{S} & \text { for } i=1, \ldots, q
\end{array}
$$

In this case, evidently, an exact pole placement for all models is not possible. However, the solution of this optimization problem stabilizes all plant models in the polytopic system and assigns approximately the closed-loop poles around the desired ones if the optimal value of $\gamma$ is small enough.

\section{B. Sensitivity Function Shaping}

In many applications, pole placement does not entirely satisfy the control specifications. It is generally needed to shape the sensitivity functions in the frequency domain to obtain certain performances and ensure some robustness with respect to unstructured uncertainty. Let us define the sensitivity function $S$, the complementary sensitivity function $T$ and the input sensitivity function $U$ as follows:

$$
\begin{aligned}
S & =(1+K G)^{-1}=M Y(M Y+N X)^{-1} \\
T & =K G(1+K G)^{-1}=N X(M Y+N X)^{-1} \\
U & =K(1+K G)^{-1}=M X(M Y+N X)^{-1}
\end{aligned}
$$

The objective is to assign the closed-loop poles in desired places and shape the sensitivity functions $S, T$ and $U$ by the weighting filters $W_{1}, W_{2}$ and $W_{3}$, respectively, such that:

$$
\left\|W_{1} S\right\|<1 \quad, \quad\left\|W_{2} T\right\|<1 \quad, \quad\left\|W_{3} U\right\|<1 .
$$

The problem is that these constraints are not convex with respect to our controller parameterization. However, the result of minimizing $\|M Y+N X-1\|_{\infty}$ is that the sensitivity functions $S, T$ and $U$ can be approximated respectively by $M Y, N X$ and $M X$ which are linear functions of $X$ and $Y$. Note that:

$$
\begin{array}{r}
\|M Y+N X-1\|_{\infty}<\gamma \Leftrightarrow|M Y+N X-1|<\gamma \quad \forall \omega \\
\Rightarrow 1-\gamma<|M Y+N X|<1+\gamma \quad \forall \omega
\end{array}
$$

Therefore:

$$
\begin{array}{ccc}
\left\|W_{1} M Y\right\|<1-\gamma & \Rightarrow & \left\|W_{1} S\right\|<1 \\
\left\|W_{1} N X\right\|<1-\gamma & \Rightarrow & \left\|W_{2} T\right\|<1 \\
\left\|W_{1} M X\right\|<1-\gamma & \Rightarrow & \left\|W_{3} U\right\|<1
\end{array}
$$

For example, for a single model $G=N M^{-1}$ if the performance specification is given by $\left\|W_{1} S\right\|_{2}<1$ and the robust stability condition with respect to unstructured uncertainty (unmodeled dynamics) by $\left\|W_{2} T\right\|_{\infty}<1$, then 
the following convex optimization problem is proposed to compute the controller:

\section{Minimize $\gamma$}

Subject to:

$$
\begin{aligned}
& \|M Y+N X-1\|_{\infty}<\gamma \\
& \left\|W_{1} M Y\right\|_{2}<1-\gamma \\
& \left\|W_{2} N X\right\|_{\infty}<1-\gamma \\
& M Y+N X \in \mathcal{S}
\end{aligned}
$$

For polytopic systems, the problem of robust pole placement with sensitivity function shaping can be presented by the following convex optimization problem:

$$
\begin{array}{ll}
\multicolumn{2}{c}{\text { Minimize } \max _{i} \gamma_{i}} \\
\text { Subject to: } \\
\left\|M_{i} Y+N_{i} X-1\right\|_{\infty}<\gamma_{i} & \text { for } i=1, \ldots, q \\
\left\|W_{1} M_{i} Y\right\|<1-\gamma_{i} & \text { for } i=1, \ldots, q \\
\left\|W_{2} N_{i} X\right\|<1-\gamma_{i} & \text { for } i=1, \ldots, q \\
\left\|W_{3} M_{i} X\right\|<1-\gamma_{i} & \text { for } i=1, \ldots, q \\
M_{i} Y+N_{i} X \in \mathcal{S} & \text { for } i=1, \ldots, q
\end{array}
$$

It is interesting to note that since the plant model parameters appear linearly in the convex constraints, the performance specifications are met not only for the vertices of the polytopic system but also for the whole polytope. In other words, the proposed method ensures robust stability and robust performance for a polytopic system.

\section{Convex Optimization}

In this section, two different methods for solving the convex optimization problem in (22) are given. In the first approach all the constraints are converted to LMI constraints, while the second approach is based on frequency gridding.

\section{A. LMI Solution}

The main point is that all transfer functions in (22), on which the norm constraints or SPRness should be applied, have known fixed denominators. These denominators are the desired closed-loop polynomial and the denominators of the weighting filters. Furthermore, the optimization variables appear linearly in the coefficients of the numerators. Thus, the use of controllable canonical state space realization $(A, B, C, D)$ for such transfer functions, leads to fixed $A$ and $B$ and linear $C$ and $D$ with respect to the optimization variables. For such state space models, norm and SPRness constraints can be converted to LMIs using the bounded real lemma and KYP lemma, respectively. In the sequel, it is shown that how an SPRness constraint or a two- or infinity-norm constraint can be represented by LMIs. These constraints are given only for the discrete-time systems. Similar LMI constraints can be obtained for continuous-time systems and these are omitted because of space limitation.
1) SPR-constraint: The SPR condition for $H_{i}=M_{i} Y+$ $N_{i} X$ can be given as an LMI, using the well-known KalmanYakubovich-Popov Lemma. For the discrete-time systems one has:

Lemma 1: (KYP lemma [18], [19]) The transfer functions $H_{i}(z)=C_{i}(z I-A)^{-1} B+D_{i}$ are SPR if and only if there exist matrices $P_{i}=P_{i}^{T}>0$ such that for $i=1, \ldots, q$ :

$$
\left[\begin{array}{cc}
A^{T} P_{i} A-P_{i} & A^{T} P_{i} B-C_{i}^{T} \\
B^{T} P_{i} A-C_{i} & B^{T} P_{i} B-D_{i}-D_{i}^{T}
\end{array}\right]<0
$$

It is evident that $A$ and $B$ (with a controllable canonical representation) are only related to the denominator of $H_{i}$ and are therefore fixed. The plant parameters for each vertex and the controller parameters (the optimization variables) appear linearly in $C_{i}$ and $D_{i}$. Hence, the above inequality becomes an LMI because the variables $P_{i}, C_{i}$ and $D_{i}$, appear affinely in the inequality. It should be mentioned that the stability condition presented by this LMI is not a quadratic stability condition since the Lyapunov type matrices $P_{i}$ are different for each vertex and related to the denominator of $H_{i}$ and not to the denominators of the plant models.

2) Norm-constraint: The following propositions are used to state the norm-constraints as LMIs for a set of transfer functions $H_{i}$, where $H_{i}$ can be $M_{i} Y+N_{i} X-1, W_{1} M_{i} Y$, $W_{2} N_{i} X$ or $W_{3} M_{i} X$. It is clear that these transfer functions are strictly proper $\left(D_{i}=0\right)$ and have fixed and known denominators. Therefore, the plant parameters appear only in $C_{i}$ in a controllable canonical realization of $H_{i}$ :

Proposition 1: Consider $H_{i}(z)$ as strictly proper stable discrete-time transfer functions with the state space realization $\left(A, B, C_{i}, D_{i}=0\right)$. Then $\left\|H_{i}\right\|_{2}<\gamma_{i}$ if and only if there exists $P_{i}=P_{i}^{T}>0$ such that for $i=1, \ldots, q$ :

$$
\begin{gathered}
{\left[\begin{array}{cc}
A^{T} P_{i} A-P_{i} & A^{T} P_{i} B \\
B^{T} P_{i} A & -\gamma_{i} I+B^{T} P_{i} B
\end{array}\right]<0} \\
{\left[\begin{array}{cc}
P_{i} & C_{i}^{T} \\
C_{i} & \gamma_{i} I
\end{array}\right]<0}
\end{gathered}
$$

Proposition 2: Consider $H_{i}(z)$ as strictly proper stable discrete-time transfer functions with the state space realization $\left(A, B, C_{i}, D_{i}=0\right)$. Then $\left\|H_{i}\right\|_{\infty}<\gamma_{i}$ if and only if there exists $P_{i}=P_{i}^{T}>0$ such that for $i=1, \ldots, q$ :

$$
\left[\begin{array}{ccc}
A^{T} P_{i} A-P & A^{T} P_{i} B & C_{i}^{T} \\
B^{T} P_{i} A & B^{T} P_{i} B-\gamma_{i} I & 0 \\
C_{i} & 0 & -\gamma_{i} I
\end{array}\right]<0
$$

Taking into account the above results, the problem (22) can be represented by LMIs and thus can be solved using efficient LMI solvers.

\section{B. Frequency Gridding}

The number of optimization variables in the LMI representation of the optimization problem in (22) is proportional to the square of the order of the closed-loop system because of the Lyapunov type matrices. Therefore, increasing the order of $X$ and $Y$ can increase significantly the number of optimization variables and may cause some numerical problems for the existing convex optimization solvers. An alternative solution is to check the constraints in some finite frequency 


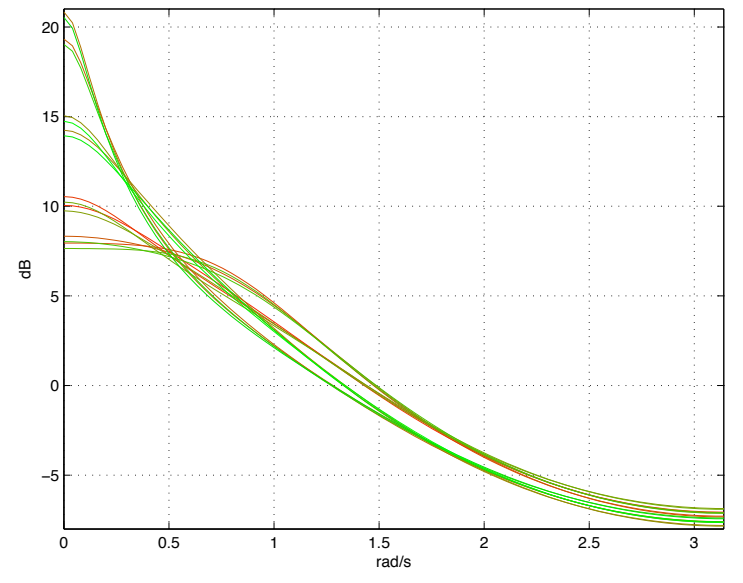

Fig. 1. Magnitude Bode diagrams of all vertices of the polytopic system.

points (frequency gridding). There are few results stating the minimum number of points to guarantee the satisfaction of the SPRness constraints [20]. Using frequency gridding, the only optimization variables are the controller parameters and the problem can be solved more efficiently. Moreover, when increasing the controller order, the optimization variables can be initialized with the values in the preceding step which leads to a faster optimization procedure. However, the results at each step should be checked out for the stability and norm constraints and if the results are not satisfactory the number of grids should be increased.

\section{Simulation Results}

Consider the problem of robust controller design for the following third-order system :

$$
G(z)=\frac{z+a}{z^{3}+b z^{2}+c z+d} \quad T_{s}=1 \mathrm{~s}
$$

with $a=-0.2, b=-1.2, c=0.5$ and $d=-0.1$ where all the parameters are uncertain up to $\pm 7 \%$ of their nominal values, resulting in a four-dimensional hypercube with $2^{4}=16$ vertices. The magnitude Bode diagrams of all the 16 vertices of the polytope are shown in Fig. 1. This figure shows that there are four groups of four models with a large uncertainty between groups at low frequencies, while the models in each group are very close to each other. It seems that the system is not an easy one for robust control design methods.

Assume that the goal is to design a controller which contains an integrator, and assign approximately the closedloop poles around the roots of

$$
P_{d}(z)=z^{2}-1.0432 z+0.3263 .
$$

The desired closed-loop poles are related to the poles of a second-order continuous-time system with a natural frequency of $0.7 \mathrm{rad} / \mathrm{s}$ and a damping factor of 0.8 (settling time $\approx 8 \mathrm{~s}$, overshoot $\approx 2 \%$ ). Furthermore, suppose that the sensitivity functions for all the systems in the polytope should satisfy $\left\|W_{1} S\right\|_{\infty}<1$, where $W_{1}(z)$ is given as follows:

$$
W_{1}(z)=\frac{0.4902\left(z^{2}-1.0432 z+0.3263\right)}{z^{2}-1.282 z+0.2821}
$$

which is a low-pass weighting filter based on the inverse of a desired sensitivity function [6]. Auxiliary poles should be much faster than the dominant poles and hence they are arbitrarily selected to be at $z=0.1$. Thus, the following coprime factorization can be chosen for the nominal plant model:

$$
\begin{aligned}
N & =\frac{z-0.2}{(z-0.1)\left(z^{2}-1.0432 z+0.3263\right)} \\
M & =\frac{z^{3}-1.2 z^{2}+0.5 z-0.1}{(z-0.1)\left(z^{2}-1.0432 z+0.3263\right)}
\end{aligned}
$$

Note that the desired dominant closed-loop poles are contained in the denominators of coprime factors of all models in the polytopic system. Since for the controllers of order less than 5 the problem becomes infeasible, a fifth-order controller $K=X Y^{-1}$ which contains an integrator is considered as follows:

$$
\begin{aligned}
& X=\frac{x_{1} z^{5}+x_{2} z^{4}+x_{3} z^{3}+x_{4} z^{2}+x_{5} z+x_{6}}{(z-0.1)^{5}} \\
& Y=\frac{(z-1)\left(y_{1} z^{4}+y_{2} z^{3}+y_{3} z^{2}+y_{4} z+y_{5}\right)}{(z-0.1)^{5}}
\end{aligned}
$$

Note that with an integrator, a third-order controller is the minimum order controller that can place the poles of only one of the 16 vertices of the system in the desired positions. Thus, a fifth-order controller is quite a reasonable order for this polytopic system.

The LMI formulation is used to solve the problem using YALMIP [21] with SeDuMi [22]. The optimal value of $\gamma=$ $\max _{i} \gamma_{i}=0.3975$ is obtained with the following controller parameters:

$$
\begin{array}{ll}
x_{1}=0.6103 & y_{1}=-0.6898 \\
x_{2}=-0.7138 & y_{2}=-0.3053 \\
x_{3}=0.3422 & y_{3}=0.06323 \\
x_{4}=-0.1249 & y_{4}=-0.07203 \\
x_{5}=0.02093 & y_{5}=0.003869 \\
x_{6}=-0.01831 &
\end{array}
$$

The closed-loop poles of all the vertices of the polytopic system are shown in Fig. 2. As can be observed, the controller brings a set of closed-loop poles of each vertex around the desired ones. The sensitivity functions of all the 16 vertices are shown in Fig. 3. The maximum value of the sensitivity functions is around $5 \mathrm{~dB}$, which is quite desirable [19]. The step responses of all vertices are shown in Fig. 4. The four groups of four models can be easily identified in the step responses. A settling time of about $12 \mathrm{~s}$ is obtained for all models while one group gives an overshoot of greater than $30 \%$. This can be explained by the fact that the model uncertainty in low frequencies is too large. However, by increasing the order of the controller, it is possible to decrease $\gamma$ and also decrease the overshoot while 


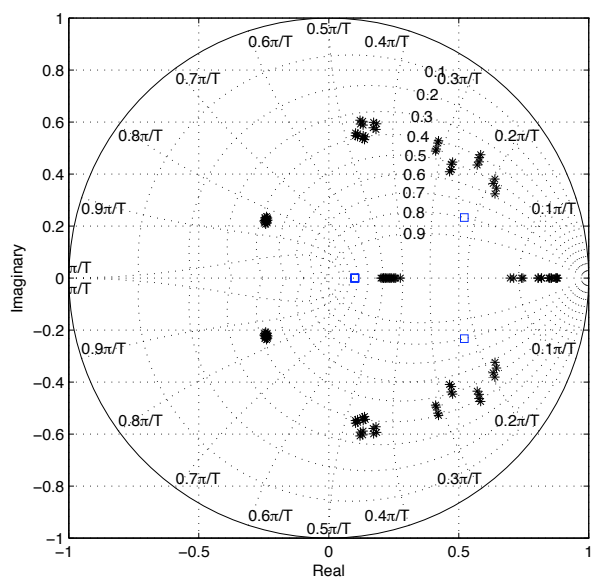

Fig. 2. Closed-loop poles of all vertices of the polytopic system with the fifth-order controller $(*)$, desired poles $(\square)$.

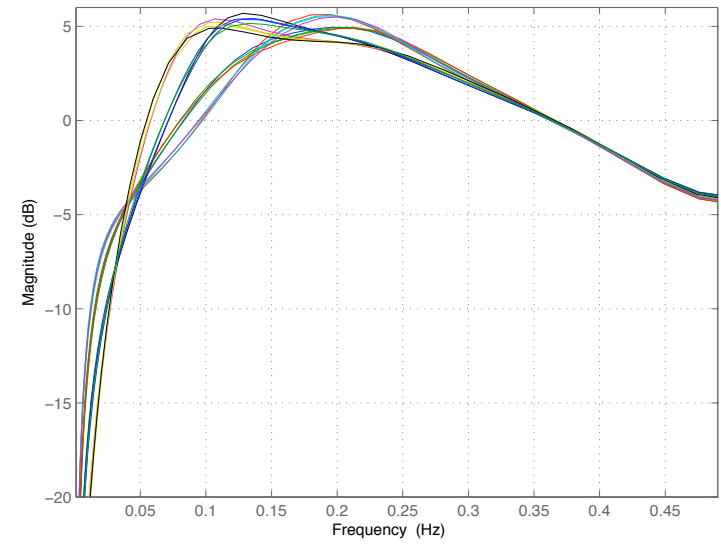

Fig. 3. Output sensitivity function of all vertices of the polytopic system with the fifth-order controller.

the maximum of output sensitivity functions remains around the same value of $5 \mathrm{~dB}$. Fig. 5 shows the step responses of all the vertices with the resulting ninth-order controller. The achieved $\gamma$ for this controller is 0.3567 and the maximum overshoot is less than $20 \%$. The interesting point is that by increasing the controller order, $\gamma$ will converge to 0.33 (see Fig. 6). So the best controller can be chosen by a tradeoff between the controller complexity and the achieved performance. It should be mentioned that increasing the controller order and consequently the number of variables, the LMI formulation could not be solved completely by SeDuMi and hence we had to use the gridding instead of LMI for controller orders greater than 7 (e.g. for an eighth-order controller we have: 3601 variables, 16 algebraic constraints and 96 matrix constraints whose maximum size is $15 \times 15$ ). With gridding, one cannot increase the controller order to more than 45 because the optimal value of $\gamma$ has already been achieved and increasing the order increases only numerical

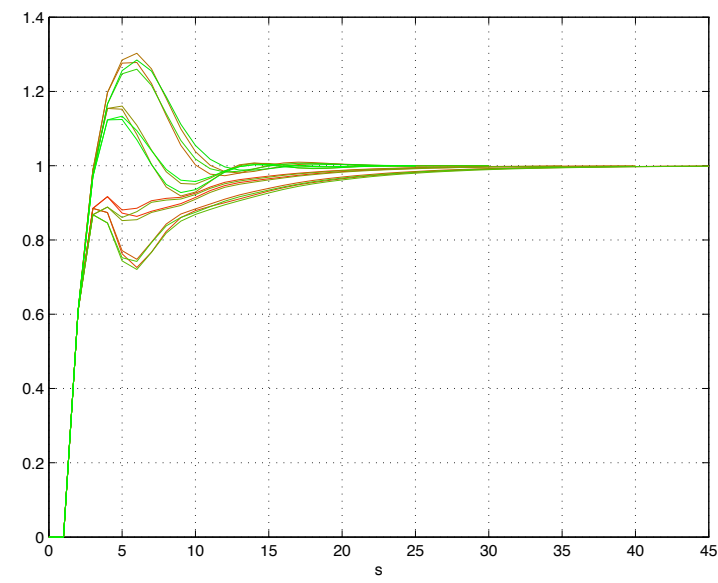

Fig. 4. Step response of all vertices of the polytopic system with the fifth-order controller

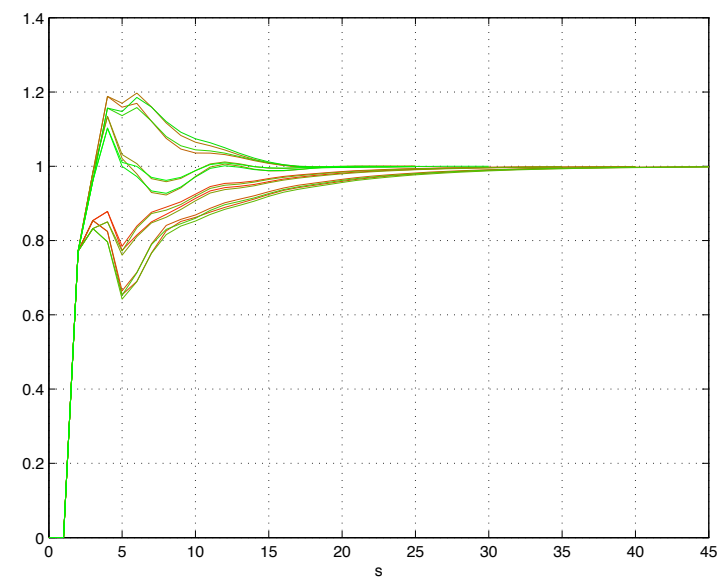

Fig. 5. Step response of all vertices of the polytopic system with the ninth-order controller.

problems.

\section{CONCLUSIONS}

A new convex parameterization of all stabilizing controllers for SISO-LTI systems based on the strict positive realness of a set of transfer functions is introduced. The main advantage of this parameterization with respect to the wellknown Youla parameterization is that it can be applied to systems with parametric uncertainty.

The approximate pole placement in the sense of the minimization of the weighted norm of the difference between the achieved and desired closed-loop characteristic polynomials is considered as performance specification. Sensitivity function shaping is considered to improve the performance of the closed-loop system and robustness with respect to the unstructured uncertainty. However, since the set of bounded weighted norm of the sensitivity functions is non-convex with respect to the new parameterization, a convex inner approximation of this set is proposed which allows the 


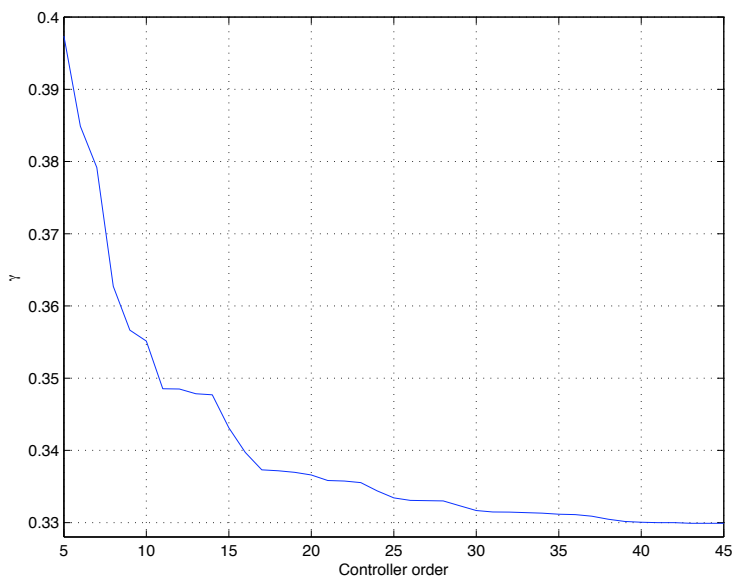

Fig. 6. $\quad \gamma$ versus controller order (gridding).

sensitivity function shaping to be performed with some conservatism.

Furthermore, the method can be used for fixed-order controller design because the controller order is independent of the model order. However, in this case, only a subset of all stabilizing fixed-order controllers are parameterized and therefore the choice of the desired closed-loop poles becomes crucial. It means that the problem of SPRness may become infeasible while there exists a stabilizing controller for the polytopic system. The related conservatism can be reduced by increasing the controller order.

\section{REFERENCES}

[1] S. P. Bhattacharyya, H. Chapellat, and L. H. Keel, Robust Control: The Parametric Approach. NJ, USA: Prentice Hall Information and System Sciences Series, 1995.

[2] B. R. Barmish, New Tools for Robustness of Linear Systems. New York, NY, 1994: Macmillan, 1994.

[3] E. Feron, P. Apkarian, and P. Gahinet, "Analysis and synthesis of robust control systems via parameter-dependent Lyapunov functions," IEEE Transactions on Automatic Control, vol. 41, no. 7, pp. 10411047, 1996.

[4] D. Peaucelle, D. Arzelier, O. Bachelier, and J. Bernussou, "A new robust D-stability condition for real convex polytopic uncertainty," Systems and Control Letters, vol. 40, pp. 21-30, 2000.

[5] G. Chesi, A. Garulli, A. Tesi, and A. Vicino, "An LMI-based technique for robust stability analysis of linear systems with polynomial parametric uncertainties," in Lecture Notes in Control and Information Sciences. Heidelberg: Springer-Verlag, 2005, vol. 312.

[6] K. Zhou, Essentials of Robust Control. New Jersey: Prentice Hall, 1998.

[7] Y. Ebihara and T. Hagiwara, "Robust controller synthesis with parameter-dependent Lyapunov variables: A dilated LMI approach," in 41st IEEE Conference on Decision and Control, Las Vegas, NV, USA, December 2002, pp. 4179-4184.

[8] P. Apkarian, H. D. Tuan, and J. Bernussou, "Continuous-time analysis, eigenstructure assignment, and $\mathrm{H}_{2}$ synthesis with enhanced linear matrix inequalities (LMI) characterizations," IEEE Transactions on Automatic Control, vol. 46, no. 12, pp. 1941-1946, December 2001.

[9] A. Rantzer and A. Megretski, "Convex parameterization of robustly stabilizing controllers," IEEE Transactions on Automatic Control, vol. 39, no. 9, pp. 1802-1808, September 1994.

[10] D. Henrion, M. Sebek, and V. Kucera, "Positive polynomials and robust stabilization with fixed-order controllers," IEEE Transactions on Automatic Control, vol. 48, no. 7, pp. 1178-1186, 2003.
[11] B. D. O. Anderson, S. Dasgupta, P. P. Khargonekar, F. J. Kraus, and M. Mansour, "Robust strict positive realness: Characterization and construction," IEEE Transactions on Circuits and Systems, vol. 37, pp. 869-876, 1990.

[12] D. Henrion, "Linear matrix inequalities for robust strictly positive real design," IEEE Transactions on Circuits and Systems I, vol. 49, no. 7, pp. 1017-1020, July 2002.

[13] G. Bianchini, A. Tesi, and A. Vicino, "Synthesis of robust strictly positive real systems with $l_{2}$ parametric uncertainty," IEEE Transactions on Circuits and Systems I, vol. 48, no. 4, pp. 438-450, April 2001.

[14] Z. S. Duan, L. Huang, and L. Wang, "Multiplier design for extended strict positive realness and its applications," International Journal of Control, vol. 77, no. 17, pp. 1493-1502, November 2004.

[15] I. D. Landau and A. Karimi, "Robust digital control using pole placement with sensitivity function shaping method," International Journal of Robust and Nonlinear Control, vol. 8, no. 2, pp. 191-210, 1998.

[16] J. Langer and I. D. Landau, "Combined pole placement and sensitivity function shaping method using convex optimization criteria," Automatica, vol. 35, pp. 1111-1120, 1999.

[17] B. Ninness and F. Gustafsson, "A unifying construction of orthonormal bases for system identification," IEEE Transactions on Automatic Control, vol. 42, no. 4, pp. 515-521, April 1997.

[18] L. Lee and J. L. Chen, "Strictly positive real lemma for discretetime descriptor systems," in 39th IEEE Conference on Decision and Control, Sydney, Australia, 2000.

[19] I. D. Landau, R. Lozano, and M. M'Saad, Adaptive Control. London: Springer-Verlag, 1997.

[20] B. D. O. Anderson, M. Mansour, and F. J. Krausp, "A new test for strict positive realness," IEEE Transactions on Circuits and Systems: Fundamental Theory and Applications, vol. 42, no. 4, pp. 226-229, April 1995.

[21] J. Löfberg, "YALMIP: A toolbox for modeling and optimization in MATLAB," in CACSD Conference, 2004. [Online]. Available: http://control.ee.ethz.ch/ joloef/yalmip.php

[22] J. F. Sturm, "Using SeDuMi 1.02, a Matlab toolbox for optimization over symmetric cones," Optimization Methods and Software, vol. 11, pp. 625-653, 1999. 